\title{
Homocysteine, interleukin-1 $\beta$, and fasting blood glucose levels as prognostic markers for diabetes mellitus complicated with cerebral infarction and correlated with carotid intima-media thickness
}

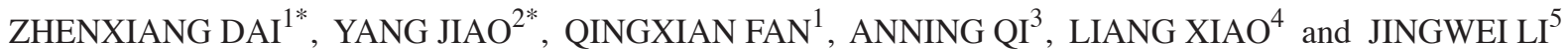 \\ Departments of ${ }^{1}$ Neurology, ${ }^{2}$ Medical Imaging, ${ }^{3}$ Laboratory Medicine and ${ }^{4}$ Emergency, \\ People's Hospital of Liuhe District of Nanjing, Nanjing, Jiangsu 211500; ${ }^{5}$ Department of \\ Neurology, Nanjing Drum Tower Hospital, Nanjing, Jiangsu 210008, P.R. China
}

Received May 8, 2019; Accepted October 2, 2019

DOI: $10.3892 /$ etm.2019.8326

\begin{abstract}
Diabetes mellitus complicated with cerebral infarction (DMCI) has a high incidence and disability rate. Therefore, identification of biomarkers for the early prediction of the development and progression of cerebral infarction (CI) is of great significance for the prevention and treatment of this disease. The roles of serum homocysteine (Hey), interleukin-1 $\beta$ (IL-1 $\beta$ ), and fasting blood glucose (FBG) in DMCI and their correlations with carotid intima-media thickness (CIMT) were explored. A total of 124 patients with DMCI (DMCI group) and 103 patients with diabetes mellitus (DM) (DM group) admitted to the People's Hospital of Liuhe District of Nanjing were enrolled in this study. A further 100 healthy controls undergoing physical examinations during the same period (HC group) were also enrolled. CIMT value was detected by carotid artery ultrasound. Hey and FBG levels were determined by a fully automatic biochemical analyzer. The IL-1 $\beta$ level was detected by enzyme-linked immunosorbent assay (ELISA). The levels of Hey, IL-1 $\beta$, and FBG and the CIMT value in the DMCI and DM groups were significantly higher than those in the HC group $(\mathrm{P}<0.001)$. The levels and the value in the DMCI group were significantly higher than those in the DM group $(\mathrm{P}<0.001)$. Hey, IL-1 $\beta$, and FBG levels were positively correlated with CIMT value $(\mathrm{r}=0.542, \mathrm{P}<0.001 ; \mathrm{r}=0.522$, $\mathrm{P}<0.001 ; \mathrm{r}=0.402, \mathrm{P}<0.001)$. Receiver operating characteristic (ROC) curves showed that the sensitivity and specificity of
\end{abstract}

Correspondence to: Dr Jingwei Li, Department of Neurology, Nanjing Drum Tower Hospital, 321 Zhongshan Road, Nanjing, Jiangsu 210008, P.R. China

E-mail: aw25x7@163.com

${ }^{*}$ Contributed equally

Key words: diabetes mellitus complicated with cerebral infarction, Hey, IL-1 $\beta$, fasting blood glucose, carotid intima-media thickness
Hey for diagnosing DMCI were 86.29 and $80.58 \%$; those of IL-1 $\beta$ were 68.55 and $86.41 \%$; those of FBG were 69.35 and $88.35 \%$. Multivariate logistic regression analysis revealed that systolic blood pressure (SBP), diastolic blood pressure (DBP), high-density lipoprotein cholesterol (HDL-C), low-density lipoprotein cholesterol (LDL-C), Hey, IL-1 $\beta$, FBG, and CIMT were independent risk factors for DMCI $(\mathrm{P}<0.05)$. In conclusion, patients with DMCI have severe atherosclerosis. Hey, IL-1 $\beta$, and FBG are involved in the development and progression of DMCI, so they can be used as predictive markers for the disease. Hey, IL-1 $\beta$, FBG, and CIMT are independent risk factors for patients with DMCI.

\section{Introduction}

Diabetes mellitus (DM) is a metabolic disease characterized by hyperglycemia, and its chronic complications can cause patient death and disability (1). There are about 415 million patients with DM worldwide, and the number has been increasing annually (2). Currently, about 193 million of the patients have not been diagnosed. Chronic DM that is untreated induces many complications and the incidence rates are still on the rise even after blood glucose is controlled (3). The long-term development of DM causes pathological changes of vascular structure, and then leads to microvascular and macrovascular complications (4). Previous findings have shown that DM is an independent risk factor for cerebral infarction (CI). The development of CI is closely related to severe metabolic dysfunction, and early CI is caused by arterial occlusion $(5,6)$. The mortality of patients with diabetes mellitus complicated with cerebral infarction (DMCI) is very high. The pathological basis of the disease is atherosclerosis and the pathological change is thrombosis (7). Therefore, the timely detection and control of macroangiopathy is of great significance to prevent and for treatment of DMCI.

Carotid intima-media thickness (CIMT) is an early manifestation of atherosclerosis, and changes in its value reflect the severity of atherosclerosis. It is practical, easy to operate, and conducive to assessing the risk of cerebrovascular diseases (8). 
Homocysteine (Hey) level plays an important role in the development and progression of DM. Its high level causes atherosclerosis and cardio-cerebrovascular diseases through damaging vascular endothelium, inducing thrombosis, and promoting the proliferation of vascular smooth muscle cells (9). DM is a chronic low-grade inflammation and is characterized by the excessive secretion of interleukin-1 $\beta$ (IL-1 $\beta$ ) and other pro-inflammatory cytokines, which enhances inflammatory signals and then results in complications such as vasculopathy $(10,11)$. Previous studies have reported that systolic blood pressure (SBP), diastolic blood pressure (DBP), high-density lipoprotein cholesterol (HDL-C), and low-density lipoprotein cholesterol (LDL-C) are major risk factors for CI (12-14). However, there are currently few studies on the diagnostic values of Hey, IL-1 $\beta$, and fasting blood glucose (FBG) levels for DMCI, or whether IL-1 $\beta$ is a risk factor for CI.

Therefore, the roles and significance of Hey, IL-1 $\beta$, and FBG levels in patients with DMCI were explored in this study.

\section{Patients and methods}

General information. Altogether 124 patients with DMCI (the DMCI group) and 103 patients with DM (the DM group) admitted to the People's Hospital of Liuhe District of Nanjing from February 2016 to January 2019 were enrolled in this study. The DMCI group consisted of 65 males and 59 females aged 46-78 years with an average age of 60.9 \pm 9.7 years. The DM group consisted of 53 males and 50 females aged 43-75 years with an average age of $60.1 \pm 9.5$ years. A further 100 healthy controls undergoing physical examinations during the same period (the HC group) were also enrolled. The group comprised 59 males and 41 females aged 39-70 years with an average age of $58.4 \pm 8.6$ years. The research subjects were informed and signed an informed consent form. This study did not violate ethics or morality and was approved by the Ethics Committee of the People's Hospital of Liuhe District (Nanjing).

Inclusion and exclusion criteria. Inclusion criteria for the study were: patients in the DMCI and DM groups met the diagnostic criteria for DM from the American Diabetes Association (ADA) in 2017 (15), and their diabetes was type II diabetes mellitus. Patients in the DMCI group met the diagnostic criteria for CI from the American Heart Association (AHA) / the American Stroke Association (ASA) in 2018 (16), and their CI was confirmed by cranial imaging, MRI, or CT. The age range of the patients in the two groups were $>38$ years, but $<78$ years, and they had complete clinical data. Exclusion criteria were: those complicated with severe hepatic and renal dysfunction, cardiogenic CI, heart failure, thyroid dysfunction, diabetic nephropathy, autoimmune diseases, severe infections, or malignant tumors; those with cognitive disorders or mental illness; those who had taken immunosuppressants or anti-inflammatory drugs in the previous one month. The inclusion and exclusion criteria were applied to the DMCI and DM groups. The healthy controls were in the HC group.

CIMT examination. Carotid artery ultrasound was performed using a Philips E33 Color Doppler Ultrasonic Diagnosis Apparatus (Koninklijke Philips Electronics N.V). All subjects were examined by the same physician. The subjects laid down for 15-min rest and then their necks were fully exposed. Next, the common carotid artery, internal carotid artery, and carotid bifurcation were successively examined at $7.5 \mathrm{MHz}$. The vertical distance between the lumen intima and the media-adventitia, which was measured at $1 \mathrm{~cm}$ before and after the carotid bifurcation, was the CIMT value. The value was measured 3 times to obtain the average value.

Detection of markers. The subjects fasted for $3 \mathrm{~h}$. In the morning the next day, their venous blood $(5 \mathrm{ml})$ was extracted, placed in vacuum blood collection tubes, and centrifuged at $1,450 \mathrm{x} \mathrm{g}$ for $10 \mathrm{~min}$ at $4^{\circ} \mathrm{C}$ with a radius of $10 \mathrm{~cm}$, so as to separate and store the upper layer of the serum for later use. The levels of serum Hey (enzymatic cycling method) and FBG (glucose oxidase method) (Beijing Bioassay Technologies Co., Ltd.; batch no. 301,401) were detected by the AU5800 fully automatic biochemical analyzer (Beckman Coulter, Inc.). The reference ranges of Hey and FBG were 5-15 $\mu \mathrm{mol} / \mathrm{l}$ and 3.33-5.55 mmol/1, respectively. The detection was carried out with reference to the instructions of the corresponding kits and instruments. Serum IL-1 $\beta$ level was detected by enzyme-linked immunosorbent assay (ELISA) (17), with steps carried out with reference to the instructions of human IL-1 $\beta$ ELISA kit [Gelatin \& Protein Co., Ltd., batch no. JK-(a)-4956]. Standard wells, sample wells to be tested, and blank control wells (without samples and enzyme-labeled reagents) were set up. The standard wells in the enzyme-labeled plate that was coated were accurately added with standard substances $(50 \mu \mathrm{l})$, while the sample wells to be tested were added with sample diluent $(40 \mu \mathrm{l})$ and then the samples to be tested $(10 \mu \mathrm{l})$ (the final dilution of the sample was 5 times). After that, the plate was coated, incubated at $37^{\circ} \mathrm{C}$ for $30 \mathrm{~min}$, and then patted dry after liquid in the wells was discarded. The plate was repeatedly washed 5 times. Each well, except the blank control wells, was added with the enzyme-labeled reagents $(50 \mu \mathrm{l})$, coated, and then incubated at $37^{\circ} \mathrm{C}$ for $30 \mathrm{~min}$. Next, each well was added with substrate A and then substrate B (50 $\mu \mathrm{l}$ each), and colored at $37^{\circ} \mathrm{C}$ for $10 \mathrm{~min}$ in the dark after the substrates were mixed well. Finally, each well was added with stop solution $(50 \mu \mathrm{l})$ to cease the reaction. Optical density (OD) values of each well were sequentially measured at $450 \mathrm{~nm}$ using a SpectraMax iD5-multifunctional microplate reader (Molecular Devices), to calculate the IL-1 $\beta$ level.

Statistical analysis. SPSS 22.0 (IBM Corp.) was used for statistical analysis. Enumeration data were expressed by the number of cases/percentage [n (\%)], and the comparison of the data between groups was analyzed by Chi-square test. Measurement data were expressed by mean \pm standard deviation (mean $\pm \mathrm{SD}$ ), and comparison of the data between groups was analyzed by independent samples t-test. One-way ANOVA was used for the comparison of means between multiple groups. LSD t-test was used for pairwise comparison between groups. Pearson's test was used to analyze the correlations of Hey, IL-1 $\beta$, and FBG levels with CIMT value. Receiver operating characteristic (ROC) curves were plotted to calculate the area under the curve (AUC), to determine the cut-off values of Hey, IL-1 $\beta$, and FBG for diagnosing DMCI, and to calculate their sensitivity and specificity. Multivariate Logistic regression was used to analyze risk factors for DMCI. P $<0.05$ indicates a statistically significant difference. 
Table I. General information $[\mathrm{n}(\%)] /($ mean $\pm \mathrm{SD})$.

\begin{tabular}{|c|c|c|c|c|c|}
\hline Categories & DMCI group $(n=124)$ & DM group $(n=103)$ & HC group $(n=100)$ & $\mathrm{t} / \mathrm{F} / \chi^{2}$ & P-value \\
\hline Sex & & & & 1.398 & 0.497 \\
\hline Male & $65(52.42)$ & $53(51.46)$ & $59(59.00)$ & & \\
\hline Female & $59(47.58)$ & $50(48.54)$ & $41(41.00)$ & & \\
\hline Age (years) & $60.9 \pm 9.7$ & $60.1 \pm 9.5$ & $58.4 \pm 8.6$ & 2.035 & 0.132 \\
\hline BMI $\left(\mathrm{kg} / \mathrm{m}^{2}\right)$ & $25.46 \pm 2.64$ & $26.04 \pm 2.49$ & $25.28 \pm 2.63$ & 2.426 & 0.090 \\
\hline Course of disease (years) & $12.3 \pm 2.5$ & $11.9 \pm 2.1$ & - & 1.289 & 0.199 \\
\hline HbAlc $(\%)$ & $10.26 \pm 2.59^{\mathrm{a}}$ & $10.12 \pm 2.96^{\mathrm{a}}$ & $4.93 \pm 0.83$ & 174.600 & $<0.001$ \\
\hline Use of insulin & & & & 0.153 & 0.696 \\
\hline Yes & $80(64.52)$ & $69(66.99)$ & - & & \\
\hline No & $44(35.48)$ & $34(33.01)$ & - & & \\
\hline History of smoking & & & & 0.643 & 0.725 \\
\hline Yes & $45(36.29)$ & $42(40.78)$ & $36(36.00)$ & & \\
\hline No & $79(63.71)$ & $61(59.22)$ & $64(64.00)$ & & \\
\hline Years of smoking & $6.8 \pm 2.7$ & $6.4 \pm 2.1$ & $6.2 \pm 1.8$ & 2.056 & 0.130 \\
\hline History of drinking & & & & 0.344 & 0.842 \\
\hline Yes & $48(38.71)$ & $43(41.75)$ & $38(38.00)$ & & \\
\hline No & $76(61.29)$ & $60(58.25)$ & $62(62.00)$ & & \\
\hline Place of residence & & & & 2.129 & 0.349 \\
\hline Yes & $91(73.39)$ & $70(67.96)$ & $77(77.00)$ & & \\
\hline No & $33(26.61)$ & $33(32.04)$ & $23(23.00)$ & & \\
\hline CHD & & & & 0.753 & 0.385 \\
\hline Yes & $11(8.87)$ & $6(5.83)$ & - & & \\
\hline No & $113(91.13)$ & 97 (94.17) & - & & \\
\hline $\mathrm{SBP}(\mathrm{mmHg})$ & $151.23 \pm 21.86^{\mathrm{a}, \mathrm{b}}$ & $143.59 \pm 21.83^{\mathrm{a}}$ & $109.52 \pm 7.15$ & 114.000 & $<0.001$ \\
\hline $\mathrm{DBP}(\mathrm{mmHg})$ & $85.73 \pm 13.67^{\mathrm{a}, \mathrm{b}}$ & $79.41 \pm 6.52^{a}$ & $76.05 \pm 8.49$ & 25.710 & $<0.001$ \\
\hline PLT (xb & $147.19 \pm 61.26$ & $146.75 \pm 71.53$ & $153.47 \pm 61.58$ & 0.349 & 0.706 \\
\hline HDL-C (mmol/l) & $1.16 \pm 0.19^{\mathrm{a}, \mathrm{b}}$ & $1.27 \pm 0.13^{\mathrm{a}}$ & $1.39 \pm 0.29$ & 10.610 & $<0.001$ \\
\hline LDL-C (mmol/l) & $3.06 \pm 0.59^{a, b}$ & $2.76 \pm 0.43^{\mathrm{a}}$ & $2.59 \pm 0.54$ & 22.910 & $<0.001$ \\
\hline $\mathrm{TC}(\mathrm{mmol} / \mathrm{l})$ & $4.63 \pm 0.83$ & $4.49 \pm 0.81$ & $4.38 \pm 0.71$ & 2.826 & 0.061 \\
\hline $\mathrm{TG}(\mathrm{mmol} / \mathrm{l})$ & $1.47 \pm 0.32^{\mathrm{a}, \mathrm{b}}$ & $1.35 \pm 0.21^{\mathrm{a}}$ & $1.23 \pm 0.57$ & 10.520 & $<0.001$ \\
\hline apoAI (g/l) & $1.01 \pm 0.19^{\mathrm{a}, \mathrm{b}}$ & $1.16 \pm 0.23^{\mathrm{a}}$ & $1.41 \pm 0.38$ & 59.850 & $<0.001$ \\
\hline apoB (g/l) & $0.98 \pm 0.32^{\mathrm{a}, \mathrm{b}}$ & $0.88 \pm 0.19^{a}$ & $0.78 \pm 0.25$ & 16.030 & $<0.001$ \\
\hline
\end{tabular}

${ }^{\mathrm{a}} \mathrm{P}<0.05$ compared with that in the HC group. ${ }^{\mathrm{b}} \mathrm{P}<0.05$ compared with that in the DM group. DMCI, diabetes mellitus complicated with cerebral infarction; BMI, body mass index; HbAlc, hemoglobin Alc; SBP, systolic blood pressure; DBP, diastolic blood pressure; PLT, platelet; HDL-C, high-density lipoprotein cholesterol; LDL-C, low-density lipoprotein cholesterol; TC, total cholesterol; TG, triglyceride; apoAI, apolipoprotein AI; apoB, apolipoprotein B.

\section{Results}

General information. There were significant differences between the DMCI, DM, and HC groups in terms of hemoglobin Alc (HbAlc), SBP, DBP, HDL-C, LDL-C, triglyceride (TG), apolipoprotein AI (apoAI), and apolipoprotein B (apoB) $(\mathrm{P}<0.05)$, but not in sex, age, body mass index (BMI), years of smoking, history of drinking, place of residence, coronary heart disease (CHD), platelet (PLT) count, and total cholesterol (TC) $(\mathrm{P}>0.05)$. There were significant differences between the DMCI and DM groups in terms of SBP, DBP, HDL-C, LDL-C, TG, apoAI, and apoB $(\mathrm{P}<0.05)$, not in course of disease, HbAlc, the use of insulin, and CHD (P>0.05) (Table I).
Levels of Hey, IL-1 $\beta$, and FBG and CIMT value. The levels of Hey, IL-1 $\beta$, and FBG and the CIMT value in the DMCI and DM groups were significantly higher than those in the HC group $(\mathrm{P}<0.001)$. The levels and the value in the DMCI group were significantly higher than those in the DM group $(\mathrm{P}<0.001)$ (Fig. 1).

Correlations of Hey, IL-1 $\beta$, and FBG levels with CIMT value in $D M C I$ group. According to the Pearson's correlation analysis, Hey level was positively correlated with CIMT value $(r=0.542$, $\mathrm{P}<0.001)$. IL-1 $\beta$ level was positively correlated with CIMT value $(\mathrm{r}=0.522, \mathrm{P}<0.001)$. FBG level was positively correlated with CIMT (r=0.402, $\mathrm{P}<0.001$ ) (Fig. 2). 
Table II. Diagnostic values of Hey, IL-1 $\beta$, and FBG levels for DMCI.

\begin{tabular}{lcccccc}
\hline Indicators & AUC & $95 \%$ CI & Standard error & Cut-off & Sensitivity (\%) & Specificity (\%) \\
\hline Hey & 0.909 & $0.872-0.945$ & 0.018 & $13.53(\mu \mathrm{mol} / \mathrm{l})$ & 86.29 & 80.58 \\
IL-1 $\beta$ & 0.842 & $0.793-0.892$ & 0.025 & $38.67(\mathrm{pg} / \mathrm{ml})$ & 68.55 & 86.41 \\
FBG & 0.837 & $0.784-0.889$ & 0.027 & $7.89(\mathrm{mmol} / \mathrm{l})$ & 69.35 & 88.35 \\
\hline
\end{tabular}

DMCI, diabetes mellitus complicated with cerebral infarction; Hey, homocysteine; IL-1 $\beta$, interleukin- $1 \beta$; FBG, fasting blood glucose.
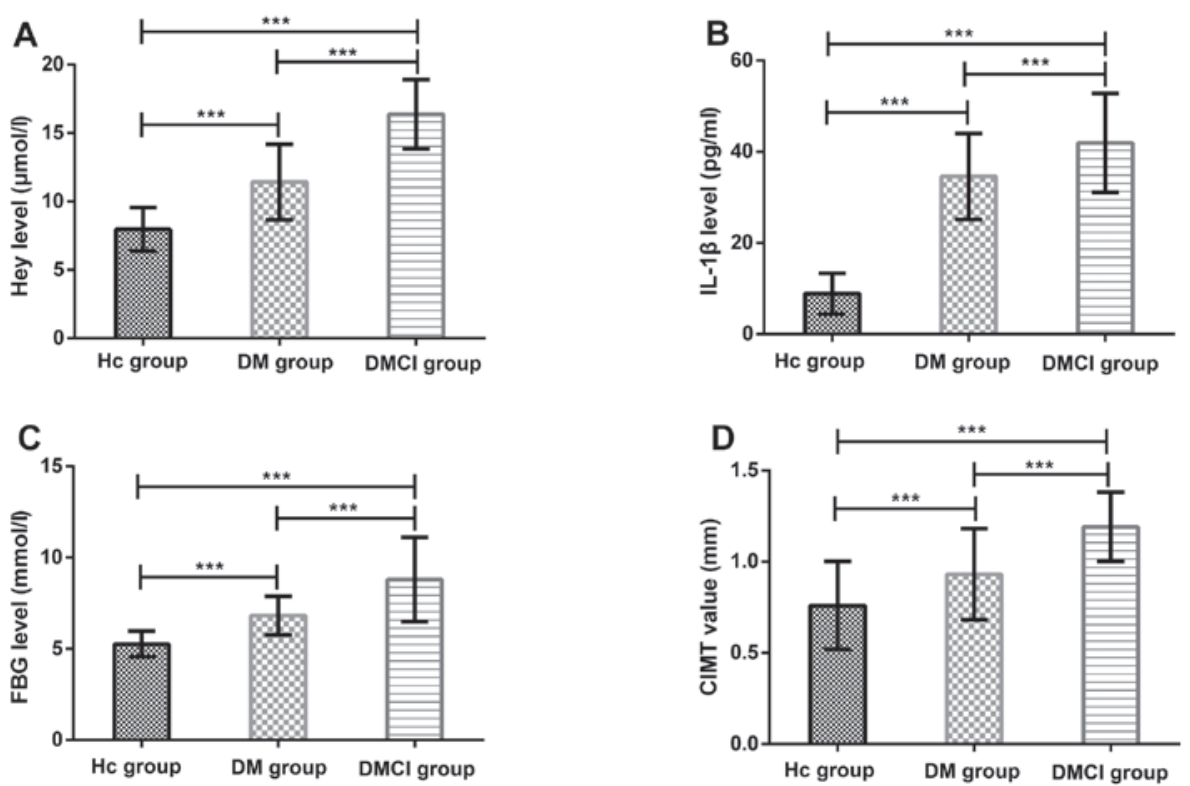

Figure 1. Comparison of levels of Hey, IL-1 $\beta$, and FBG and CIMT value. (A) Comparison of Hey level between the HC, DM, and DMCI groups. (B) Comparison of IL-1 $\beta$ level between the HC, DM, and DMCI groups. (C) Comparison of FBG level between the HC, DM, and DMCI groups. (D) Comparison of CIMT value between the HC, DM, and DMCI groups. ${ }^{* * *} \mathrm{P}<0.001$. HC, healthy controls; DM, diabetes mellitus; DMCI, diabetes mellitus complicated with cerebral infarction; Hey, homocysteine; IL-1 $\beta$, interleukin-1 $\beta$; FBG, fasting blood glucose; CIMT, carotid intima-media thickness.
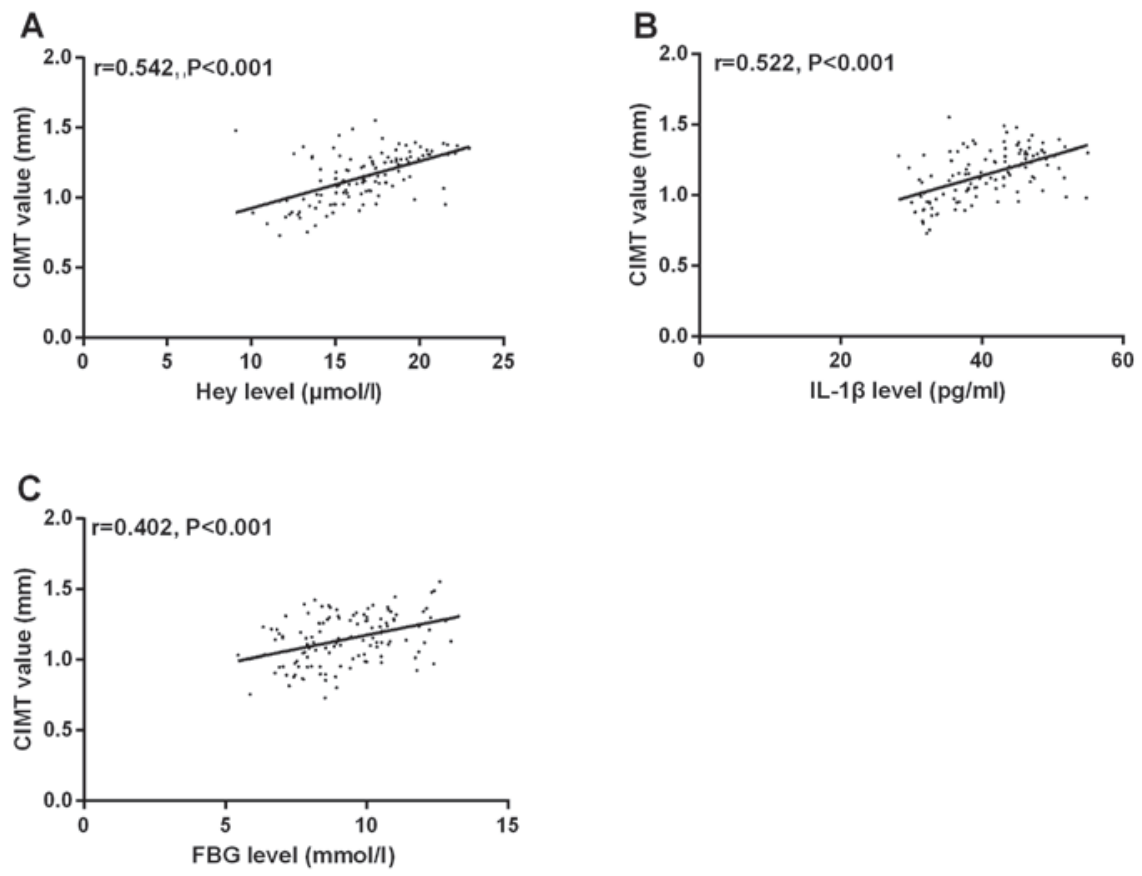

Figure 2. Correlation of Hey, IL-1 $\beta$, and FBG levels with CIMT value in DMCI group. (A) Correlation of Hey level with CIMT value in the DMCI group. (B) Correlation of IL-1 $\beta$ level with CIMT value in the DMCI group. (C) Correlation of FBG level with CIMT value in the DMCI group. Hey, homocysteine; DMCI, diabetes mellitus complicated with cerebral infarction; IL-1 $\beta$, interleukin-1 $\beta$; FBG, fasting blood glucose; CIMT, carotid intima-media thickness. 
A
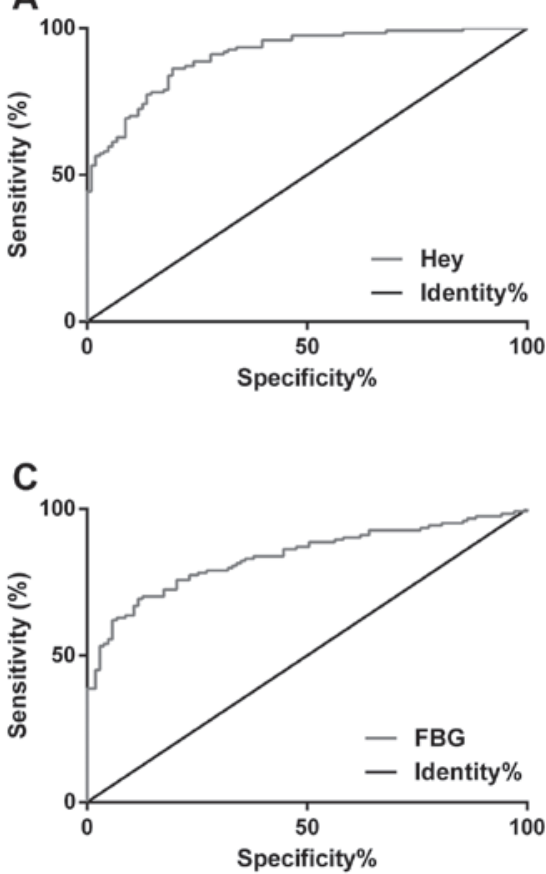

B

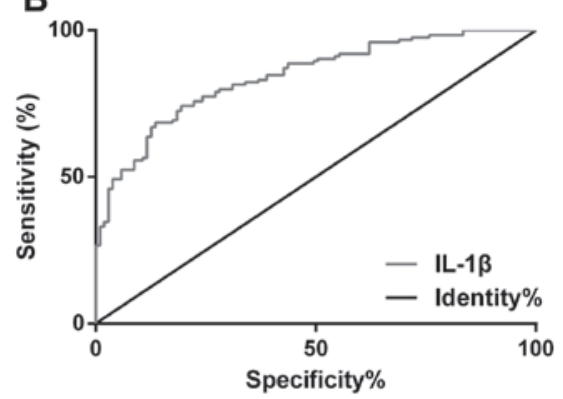

Figure 3. ROC curves of Hey, IL-1 $\beta$, and FBG levels for diagnosing DMCI. (A) ROC curve of Hey level for diagnosing DMCI. (B) ROC curve of IL-1 $\beta$ level for diagnosing DMCI. (C) ROC curve of FBG level for diagnosing DMCI. ROC, receiver operating characteristic; DMCI, diabetes mellitus complicated with cerebral infarction; Hey, homocysteine; IL-1 $\beta$, interleukin-1 $\beta$; FBG, fasting blood glucose.

Table III. Assignment of multivariate Logistic regression analysis.

\begin{tabular}{lcc}
\hline Factors & Variables & Assignment \\
\hline SBP $(\mathrm{mmHg})$ & X1 & A continuous variable \\
DBP $(\mathrm{mmHg})$ & X2 & A continuous variable \\
HDL-C $(\mathrm{mmol} / \mathrm{l})$ & $\mathrm{X} 3$ & A continuous variable \\
LDL-C $(\mathrm{mmol} / \mathrm{l})$ & $\mathrm{X} 4$ & A continuous variable \\
TG $(\mathrm{mmol} / \mathrm{l})$ & $\mathrm{X} 5$ & A continuous variable \\
apoAI $(\mathrm{g} / \mathrm{l})$ & $\mathrm{X} 6$ & A continuous variable \\
apoB $(\mathrm{g} / \mathrm{l})$ & $\mathrm{X} 7$ & A continuous variable \\
Hey $(\mu \mathrm{mol} / \mathrm{l})$ & $\mathrm{X} 8$ & A continuous variable \\
$\mathrm{IL}-1 \beta(\mathrm{pg} / \mathrm{ml})$ & $\mathrm{X} 9$ & A continuous variable \\
FBG $(\mathrm{mmol} / \mathrm{l})$ & $\mathrm{X} 10$ & A continuous variable \\
CIMT $(\mathrm{mm})$ & $\mathrm{X} 11$ & A continuous variable
\end{tabular}

SBP, systolic blood pressure; DBP, diastolic blood pressure; HDL-C, high-density lipoprotein cholesterol; LDL-C, low-density lipoprotein cholesterol; TG, triglyceride; apoAI, apolipoprotein AI; apoB, apolipoprotein B; Hey, homocysteine; IL-1 $\beta$, interleukin-1 $\beta$; FBG, fasting blood glucose; CIMT, carotid intima-media thickness.

Diagnostic values of Hey, IL-1 $\beta$, and FBG levels for DMCI. The ROC curves of Hey, IL-1 $\beta$, and FBG levels for diagnosing DMCI were plotted. The AUC of Hey for the diagnosis was 0.909 (95\% CI, 0.872-0.945) and the cut-off value was $13.53 \mu \mathrm{mol} / \mathrm{l}$, with the sensitivity of 86.29 and the specificity of $80.58 \%$. The AUC of IL- $1 \beta$ was 0.842 (95\% CI, 0.793-0.892) and the cut-off value was $38.67 \mathrm{pg} / \mathrm{ml}$, with the sensitivity of 68.55 and the specificity of $86.41 \%$. The AUC of FBG was 0.837 (95\% CI, 0.784-0.889) and the cut-off value was $7.89 \mathrm{pg} / \mathrm{ml}$, with the sensitivity of 69.35 and the specificity of $88.35 \%$ (Table II and Fig. 3).

Multivariate logistic regression analysis of risk factors for $D M C I$. The multivariate Logistic regression analysis was performed on factors with differences between the DMCI and DM groups. The results showed that SBP $(\mathrm{P}=0.007)$, DBP $(\mathrm{P}=0.009)$, HDL-C $(\mathrm{P}=0.001)$, LDL-C $(\mathrm{P}=0.041)$, Hey $(\mathrm{P}<0.001)$, IL-1 $\beta(\mathrm{P}=0.003), \mathrm{FBG}(\mathrm{P}=0.049)$, and CIMT $(\mathrm{P}=0.006)$ were independent risk factors for DMCI $(\mathrm{P}<0.05)$ (Tables III and IV).

\section{Discussion}

$\mathrm{DM}$ is the main cause of macroangiopathy and its incidence has been increasing year by year. With the improvement of living standards and the development of economy, increasing number of young people have developed DM (18). The pathological basis of CI is atherosclerotic plaques, which lead to luminal stenosis, then microthrombus, and finally hypoxia and ischemia of the brain tissue (19). As a long-term chronic result, DMCI has no obvious clinical signs and symptoms during this period (20). Therefore, to diagnose DMCI in time and analyze its risk factors is of great significance.

CIMT predicts the risk of coronary artery stenosis in asymptomatic patients. Related to the severity of atherosclerosis, its value is significantly higher in patients with type II DM than that in healthy people (21). As an intermediate metabolite of sulfur amino acids, Hey activates the coagulation system and promotes deposition on vascular walls, thus affecting vasodilation (22). IL-1 $\beta$ is a pro-inflammatory cytokine that plays an important role during atherothrombosis. 
Table IV. Multivariate logistic regression analysis of risk factors for DMCI.

\begin{tabular}{llcrcc}
\hline Factors & \multicolumn{1}{c}{ B } & Standard error & Wals & P-value & HR (95\% CI) \\
\hline SBP $(\mathrm{mmHg})$ & 0.127 & 0.047 & 7.362 & 0.007 & $1.135(1.036-1.245)$ \\
DBP $(\mathrm{mmHg})$ & 0.062 & 0.024 & 6.865 & 0.009 & $1.064(1.016-1.115)$ \\
HDL-C $(\mathrm{mmol} / \mathrm{l})$ & -6.458 & 1.988 & 10.555 & 0.001 & $0.002(0.000-0.077)$ \\
LDL-C $(\mathrm{mmol} / \mathrm{l})$ & 1.16 & 0.687 & 2.852 & 0.041 & $3.191(0.830-12.267)$ \\
TG $(\mathrm{mmol} / \mathrm{l})$ & 1.473 & 1.184 & 1.549 & 0.213 & $4.363(0.429-44.394)$ \\
apoAI $(\mathrm{g} / \mathrm{l})$ & -2.839 & 1.67 & 2.890 & 0.089 & $0.058(0.002-1.543)$ \\
apoB $(\mathrm{g} / \mathrm{l})$ & 0.359 & 0.210 & 2.930 & 0.087 & $1.432(0.949-2.159)$ \\
Hey $(\mu \mathrm{mol} / \mathrm{l})$ & 0.788 & 0.159 & 24.718 & $<0.001$ & $2.199(1.612-3.001)$ \\
IL-1 $(\mathrm{pg} / \mathrm{ml})$ & 0.163 & 0.054 & 9.031 & 0.003 & $1.177(1.058-1.309)$ \\
FBG $(\mathrm{mmol} / \mathrm{l})$ & 0.311 & 0.161 & 3.721 & 0.049 & $1.364(0.995-1.871)$ \\
CIMT $(\mathrm{mm})$ & 4.267 & 1.563 & 7.456 & 0.006 & $71.281(3.333-1524.23)$ \\
\hline
\end{tabular}

DMCI, diabetes mellitus complicated with cerebral infarction; systolic SBP, systolic blood pressure; DBP, diastolic blood pressure; HDL-C, high-density lipoprotein cholesterol; LDL-C, low-density lipoprotein cholesterol; TG, triglyceride; apoAI, apolipoprotein AI; apoB, apolipoprotein B; Hey, homocysteine; IL-1 $\beta$, interleukin-1 $\beta$; FBG, fasting blood glucose; CIMT, carotid intima-media thickness.

IL-1 $\beta$-mediated inflammation functions in the deterioration of islet $\beta$ cell function, atherogenesis, and plaque progression (23). In this study, the levels of Hey, IL-1 $\beta$, and FBG and the CIMT value in the DMCI and DM groups were significantly higher than those in the HC group; the levels and the value in the DMCI group were significantly higher than those in the DM group. This indicates that patients with DMCI have severe atherosclerosis, and that Hey, IL-1 $\beta$, and FBG are involved in the development and progression of DMCI. Moreover, Hey, IL-1 $\beta$, and FBG levels were positively correlated with CIMT value, which shows that the three levels may affect CIMT, so Hey, IL-1 $\beta$, and FBG are expected to be indicators for evaluating the severity of DMCI. In a study by Matsumoto et al (24), the CIMT value in the DMCI group is significantly higher than that in the CI group. The probability of CI in patients with DM increases by 1.8 times every time CIMT increases by $0.1 \mathrm{~mm}$. HbAlc and age are independently related to CIMT. In a study by Eikelboom et al (25), Hey is closely associated with the pathological changes of arteries, and its level is positively correlated with vascular stenosis and arteriosclerosis, so high Hey level is an independent risk factor for CI. In a study by Huang et al (26), large glucose fluctuations are related to $\mathrm{CI}$ and the poor short-term prognosis of patients with the disease. The results of this study showed that SBP, DBP, HDL-C, LDL-C, Hey, FBG, and CIMT were independent risk factors for DMCI, which is similar to the findings of previous studies (27-29). Whether IL-1 $\beta$ can be used as an independent risk factor for DMCI has been rarely studied. According to a previous study, IL-1 $\beta$ release in the cerebral cortex of Fischer rats increases after permanent middle cerebral artery occlusion, while blocking IL- $1 \beta$ with anti-IL-1 $\beta$ antibody reduces the scope of CI, and relieves neurological and behavioral dysfunction (30). Thus, IL-1 $\beta$ may play an important role in CI. The results of the present study showed that IL-1 $\beta$ was an independent risk factor for DMCI. The massive release of Hey damages the vascular endothelial function and unbalances the coagulation and fibrinolytic systems, thereby causing a prethrombotic state (31). Vascular inflammatory responses accelerate the formation of unstable plaques. Hy perglycemia causes acidosis of brain cells through lactic acid accumulation during cerebral ischemia. It also causes brain cell damage through the destruction of mitochondrial function, the promotion of free radical generation, and the enhancement of lipid peroxidation $(32,33)$. Therefore, the development and progression of DMCI may be the result of synergy between Hey, IL-1 $\beta$, and hyperglycemia. There are currently few studies on the diagnostic values of Hey, IL-1 $\beta$, and FBG levels for DMCI. According to the ROC curves in this study, the three levels had good predictive values for DMCI, and the cut-off values of the three for diagnosing the disease were $13.53 \mu \mathrm{mol} / 1$, $38.67 \mathrm{pg} / \mathrm{ml}$, and $7.89 \mathrm{mmol} / 1$, respectively. The observation of the cut-off values was helpful to identify DM and DMCI. Therefore, Hey, IL-1 $\beta$, and FBG levels can be monitored and intervened, which is conducive to reducing cervical atherosclerosis, delaying the progression of atherosclerosis, and preventing DMCI.

This study confirms the roles of Hey, IL-1 $\beta$, and FBG in the development and progression of DMCI, but it still has deficiencies. The changes in the three levels during treatment were not observed. Basic experiments were not carried out. The regulatory mechanisms of Hey, IL- $1 \beta$, and FBG in the development and progression of DMCI were not fully explored. These deficiencies need to be supplemented in future studies to corroborate the conclusions of this study.

In summary, patients with DMCI have severe atherosclerosis. Hey, IL-1 $\beta$, and FBG are involved in the development and progression of DMCI, so they can be used as predictive markers for the disease. Hey, IL-1 $\beta$, FBG, and CIMT are independent risk factors for patients with DMCI.

\section{Acknowledgements}

Not applicable. 


\section{Funding}

This study was supported by Value of Grass-roots Hospital's Green Channel in the Treatment of Acute Cerebral Infarction of The Project Fund of Nanjing Health Committee (no. YKK17241).

\section{Availability of data and materials}

The datasets used and/or analyzed during the present study are available from the corresponding author on reasonable request.

\section{Authors' contributions}

$\mathrm{ZD}$ was the major contributor in writing the manuscript and carried out all experiments. YJ analyzed and interpreted the patient general data. QF and AQ performed ELISA. LX and JL were responsible for analysis of the observation indicators. All the authors read and approved the final manuscript.

\section{Ethics approval and consent to participate}

The study was approved by the Ethics Committee of the People's Hospital of Liuhe District of Nanjing. Patients who participated in this research, signed an informed consent and had complete clinical data.

\section{Patient consent for publication}

Not applicable.

\section{Competing interests}

The authors declare that they have no competing interests.

\section{References}

1. Setoodeh A, Didban A, Rabbani A, Sayarifard A, Abbasi F, Sayarifard F and Hoseinzade F: The effect of metformin as an adjunct therapy in adolescents with type 1 diabetes. J Clin Diagn Res 11: SC01-SC04, 2017.

2. Chawla A, Chawla R and Jaggi S: Microvasular and macrovascular complications in diabetes mellitus: Distinct or continuum? Indian J Endocrinol Metab 20: 546-551, 2016.

3. Patel A, MacMahon S, Chalmers J, Neal B, Billot L, Woodward M, Marre M, Cooper M, Glasziou P, Grobbee D, et al; ADVANCE Collaborative Group: Intensive blood glucose control and vascular outcomes in patients with type 2 diabetes. N Engl J Med 358: 2560-2572, 2008

4. Wu D, Wu C and Zhong Y: The association between paraoxonase 1 activity and the susceptibilities of diabetes mellitus, diabetic macroangiopathy and diabetic microangiopathy. J Cell Mol Med 22: 4283-4291, 2018

5. Güven A, Hancili S, Karatoprak EY and Tasel B: Symptomatic cerebral infarction in a child with severe diabetic ketoacidosis. J Pediatr Endocrinol Metab 27: 1001-1004, 2014.

6. Zhao L and Hu FX: $\alpha$-Lipoic acid treatment of aged type 2 diabetes mellitus complicated with acute cerebral infarction. Eur Rev Med Pharmacol Sci 18: 3715-3719, 2014.

7. Zhang B, Wang D, Ji TF, Shi L and Yu JL: Overexpression of lncRNA ANRIL up-regulates VEGF expression and promotes angiogenesis of diabetes mellitus combined with cerebral infarction by activating NF- $\mathrm{BB}$ signaling pathway in a rat model. Oncotarget 8: 17347-17359, 2017.

8. Wang SW, Liu Z and Shi ZS: Non-coding RNA in acute ischemic stroke: Mechanisms, biomarkers and therapeutic targets. Cell Transplant 27: 1763-1777, 2018.
9. Lin T, Liu JC, Chang LY and Shen CW: Association of C-reactive protein and homocysteine with subclinical coronary plaque subtype and stenosis using low-dose MDCT coronary angiography. Atherosclerosis 212: 501-506, 2010.

10. Konya H, Miuchi M, Satani K, Matsutani S, Yano Y, Tsunoda T, Ikawa T, Matsuo T, Ochi F, Kusunoki Y, et al: Asymmetric dimethylarginine, a biomarker of cardiovascular complications in diabetes mellitus. World J Exp Med 5: 110-119, 2015.

11. Glaser NS, Ghetti S, Casper TC, Dean JM and Kuppermann N; Pediatric Emergency Care Applied Research Network (PECARN) DKA FLUID Study Group: Pediatric diabetic ketoacidosis, fluid therapy, and cerebral injury: The design of a factorial randomized controlled trial. Pediatr Diabetes 14: 435-446, 2013.

12. Fan X, Lo EH and Wang X: Effects of minocycline plus tissue plasminogen activator combination therapy after focal embolic stroke in type 1 diabetic rats. Stroke 44: 745-752, 2013.

13. Mohan V, Cooper ME, Matthews DR and Khunti K: The standard of care in type 2 diabetes: Re-evaluating the treatment paradigm. Diabetes Ther 10 (Suppl 1): 1-13, 2019.

14. Osei E, den Hertog HM, Berkhemer OA, Fransen PS, Roos YB, Beumer D, van Oostenbrugge RJ, Schonewille WJ, Boiten J, Zandbergen AA, et al; MR CLEAN pretrial investigators: Increased admission and fasting glucose are associated with unfavorable short-term outcome after intra-arterial treatment of ischemic stroke in the MR CLEAN pretrial cohort. J Neurol Sci 371: 1-5, 201, 2016.

15. American Diabetes Association: 8. Pharmacologic approaches to glycemic treatment: Standards of Medical Care in Diabetes-2018. Diabetes Care 41 (Suppl 1): S73-S85, 2018.

16. Powers WJ, Rabinstein AA, Ackerson T, Adeoye OM, Bambakidis NC, Becker K, Biller J, Brown M, Demaerschalk BM, Hoh B, et al; American Heart Association Stroke Council: 2018 guidelines for the early management of patients with acute ischemic stroke: A guideline for healthcare professionals from the American Heart Association/American Stroke Association. Stroke 49: e46-e110, 2018.

17. Charrad R, Berraïes A, Hamdi B, Ammar J, Hamzaoui K and Hamzaoui A: Anti-inflammatory activity of IL-37 in asthmatic children: Correlation with inflammatory cytokines TNF- $\alpha$, IL- $\beta$, IL-6 and IL-17A. Immunobiology 221: 182-187, 2016.

18. Joung B, Lee JM, Lee KH, Kim TH, Choi EK, Lim WH, Kang KW, Shim J, Lim HE, Park J, et al; KHRS Atrial Fibrillation Guideline Working Group: 2018 Korean guideline of atrial fibrillation management. Korean Circ J 48: 1033-1080, 2018.

19. Liu L, Ding J, Leng X, Pu Y, Huang LA, Xu A, Wong KSL, Wang $X$ and Wang Y: Guidelines for evaluation and management of cerebral collateral circulation in ischaemic stroke 2017. Stroke Vasc Neurol 3: 117-130, 2018.

20. McCoy CE, Langdorf MI and Lotfipour S: American Heart Association/American Stroke Association Deletes Sections from 2018 Stroke Guidelines. West J Emerg Med 19: 947-951, 2018.

21. Sun YP, Cai YY, Li HM, Deng SM, Leng RX and Pan HF: Increased carotid intima-media thickness (CIMT) levels in patients with type 1 diabetes mellitus (T1DM): A meta-analysis. J Diabetes Complications 29: 724-730, 2015.

22. Neumiller JJ and Umpierrez GE: 2018 Standards of care update: Pharmacologic approaches to glycemic management in people with type 2 diabetes. Diabetes Spectr 31: 254-260, 2018

23. American Diabetes Association: Standards of medical care in diabetes-2018 abridged for primary care providers. Clin Diabetes 36: 14-37, 2018.

24. Matsumoto K, Sera Y, Nakamura H, Ueki Y and Miyake S: Correlation between common carotid arterial wall thickness and ischemic stroke in patients with type 2 diabetes mellitus. Metabolism 51: 244-247, 2002.

25. Eikelboom JW, Hankey GJ, Anand SS, Lofthouse E, Staples N and Baker RI: Association between high homocyst(e)ine and ischemic stroke due to large- and small-artery disease but not other etiologic subtypes of ischemic stroke. Stroke 31: 1069-1075, 2000.

26. Huang J, Zhang X, Li J, Tang L, Jiao X and Lv X: Impact of glucose fluctuation on acute cerebral infarction in type 2 diabetes. Can J Neurol Sci 41: 486-492, 2014.

27. Yang N, Lin M, Wang BG, Zeng WY, He YF, Peng HY, Zeng J, Wu ZY and Zhong Y: Low level of low-density lipoprotein cholesterol is related with increased hemorrhagic transformation after acute ischemic cerebral infarction. Eur Rev Med Pharmacol Sci 20: 673-678, 2016 
28. You S, Zhong C, Xu J, Han Q, Zhang X, Liu H, Zhang Y, Shi J, Huang Z, Xiao G, et al: LDL-C/HDL-C ratio and risk of all-cause mortality in patients with intracerebral hemorrhage. Neurol Res 38: 903-908, 2016

29. Zhang XL, Fu HJ, Yang GR, Wan G, Li D, Zhu LX, Xie RR, Lv YJ, Zhang JD, Li YL, et al; Beijing Communities Diabetes Study Group: The effects of cardiovascular risk factor combined anti-platelet therapy and the risk of cerebrovascular events in patients with T2DM in an urban community over 96-months follow-up: The Beijing communities diabetes study 19. Diabetes Res Clin Pract 144: 236-244, 2018.

30. Kirkman MS, Mahmud H and Korytkowski MT: Intensive blood glucose control and vascular outcomes in patients with type 2 diabetes mellitus. Endocrinol Metab Clin North Am 47: 81-96, 2018.
31. Wu W, Guan Y, Xu K, Fu XJ, Lei XF, Lei LJ, Zhang ZQ, Cheng Y and Li YQ: Plasma homocysteine levels predict the risk of acute cerebral infarction in patients with carotid artery lesions. Mol Neurobiol 53: 2510-2517, 2016.

32. Fayfman M, Pasquel FJ and Umpierrez GE: Management of hyperglycemic crises: Diabetic ketoacidosis and hyperglycemic hyperosmolar state. Med Clin North Am 101: 587-606, 2017.

33. Olamoyegun M, Ibraheem W, Iwuala S, Audu M and Kolawole B: Burden and pattern of micro vascular complications in type 2 diabetes in a tertiary health institution in Nigeria. Afr Health Sci 15: 1136-1141, 2015

(i) $(-)$ This work is licensed under a Creative Commons Attribution-NonCommercial-NoDerivatives 4.0 International (CC BY-NC-ND 4.0) License. 